\title{
Experiences of Patient-Centered Medical Home Staff Team Members Working in Interprofessional Training Environments
}

Summer Newell, $P h D, M P H^{7}$ (D) Bridget O'Brien, $P h D^{2,3}$, Rebecca Brienza, MD, MPH ${ }^{4,5}$,

Maya Dulay, MD ${ }^{2,3}$, Anna Strewler, MS, AGPCNP-BC ${ }^{2,6,7}$, Jennifer K. Manuel, $P h D^{2,8}$, and Anaïs Tuepker, $\mathrm{PhD}, \mathrm{MPH}^{1,9}$

'Center to Improve Veteran Involvement in Care (CIVIC), VA Portland Healthcare System Portland, OR, USA; ${ }^{2}$ VA San Francisco Healthcare System San Francisco, CA, USA; ${ }^{3}$ University of California, San Francisco School of Medicine San Francisco, CA, USA; ${ }^{4}$ VA Connecticut Healthcare System West Haven, CT, USA; ${ }^{5}$ Yale School of Medicine New Haven, CT, USA; 6 University of California, San Francisco School of Nursing San Francisco, CA, USA; ${ }^{7}$ Department of Community Health Systems, University of California, San Francisco San Francisco, CA, USA; ${ }^{8}$ Department of Psychiatry, University of California San Francisco, CA, USA; ${ }^{9}$ Division of General Internal Medicine, Oregon Health \& Science University Portland, OR, USA.

BACKGROUND: Evidence is growing that interprofessional team-based models benefit providers, trainees, and patients, but less is understood about the experiences of staff who work beside trainees learning these models.

OBJECTIVE: To understand the experiences of staff in five VA training clinics participating in an interprofessional team-based learning initiative.

DESIGN: Individual semi-structured interviews with staff were conducted during site visits, qualitatively coded, and analyzed for themes across sites and participant groups.

PARTICIPANTS: Patient-centered medical home (PCMH) staff members ( $n=32$; RNs, Clinical and Clerical Associates) in non-primary care provider (PCP) roles working on teams with trainees from medicine, nursing, pharmacy, and psychology.

APPROACH: Benefits and challenges of working in an interprofessional, academic clinic were coded by the primary author using a hybrid inductive/directed thematic analytic approach, with review and iterative theme development by the interprofessional author team.

KEY RESULTS: Efforts to improve interprofessional collaboration among trainees and providers, such as increased shared leadership, have positive spillover effects for PCMH staff members. These staff members perceive themselves playing an educational role for trainees that is not always acknowledged. Playing this role, learning from the "fresh" knowledge imparted by trainees, and contributing to the future of health care all bring satisfaction to staff members. Some constraints exist for full participation in the educational efforts of the clinic.

CONCLUSIONS: Increased recognition of and expanded support for PCMH staff members to participate in educational endeavors is essential as interprofessional training clinics grow.

KEY WORDS: health care workforce; interprofessional education; interprofessional practice; patient-centered medical home; academic clinic; qualitative methods.

Electronic supplementary material The online version of this article (https://doi.org/10.1007/s11606-020-06055-y) contains supplementary material, which is available to authorized users.

Received December 7, 2019

Accepted July 13, 2020

Published online July 29, 2020
J Gen Intern Med 35(10):2976-82

DOI: $10.1007 / \mathrm{s} 11606-020-06055-\mathrm{y}$

(c) Society of General Internal Medicine (This is a U.S. government work and not under copyright protection in the U.S.; foreign copyright protection may apply) 2020

\section{INTRODUCTION}

In the last decade, there has been an increasingly loud and consistent call for the next generation of health care workers to be trained to work effectively in patient-centered medical home (PCMH) settings. ${ }^{1-4}$ In the PCMH model, Nurse Care Managers (RNs), Clinical Associates (LVNs/LPNs), and Administrative/ Clerical staff, among others, take on expanded roles $^{5}$ working together with the team on tasks including panel and population management and (co)leading team huddles with physicians and other providers. ${ }^{6}$ Interprofessional, team-based practice has been found to be a "building block" of high performing primary care ${ }^{7}$ and the team-based skills of staff members in these practices may range from expert to novice. The potential for all PCMH staff members to influence trainee learning has been recognized, leading to recommendations that team staff be given resources to develop and support their evolving teaching roles. ${ }^{8,}{ }^{9}$ Rarely are these care team members formally viewed as teaching faculty by leadership, nor are they considered potential learners who might benefit from the trainee-focused educational activities occurring on their teams. Existing literature on interprofessional clinical education has primarily focused on outcomes for trainees and faculty, with less attention paid to outcomes for non-faculty staff members. This study examined how working with trainees impacts the experiences and satisfaction among non-faculty staff members, as well as the role played by all team members in preparing trainees for collaborative practice.

\section{METHODS}

\section{Setting}

The Department of Veterans Affairs (VA) conducts the largest education and training effort for health professionals in the 
USA. ${ }^{10}$ In 2011, VA invested in five Centers of Excellence in Primary Care Education (CoEPCE) dedicated to integrating interprofessional trainees into VA's patient-centered medical home (PCMH) model called Patient-Aligned Care Teams (PACT). ${ }^{11}$ All VA medical facilities and their academic affiliates $^{12}$ were invited to respond to a national request for proposals. Selection criteria included quality of VA PACT implementation to date, capacity to expand training in the PACT setting, commitment to interprofessional care and education, and proposed strategies for developing educational leadership, curriculum, and evaluation to advance the project goals. Five CoEPCE sites were selected for funding.

While VA PACT implementation focused on the transformation of clinical care, the CoEPCE initiative had a distinct but complementary focus on redesign and integration of health professions education and improved alignment between educational and clinical initiatives in primary care settings. ${ }^{13,14}$

\section{Participants}

As part of a mixed-methods evaluation of the original five CoEPCEs, an evaluation team (comprising clinicians and social scientists funded and based outside of the participating sites) interviewed non-faculty staff members working on teams with trainees from medicine, nursing, pharmacy, and psychology. We aimed for a minimum of one interview per role type (RNs, Clerical Associates, Clinical Associates) per site. Interviews were voluntary and were conducted with any available staff who agreed to be interviewed in-person during a site. Interviews followed a semi-structured interview guide and were conducted and audio recorded by one of three interviewers (including the first and last author) with graduate level training in qualitative methods. Questions focused on perceived advantages and disadvantages of working with trainees and impacts on professional engagement and satisfaction (See Appendix 1).

\section{Analysis}

Interviews were transcribed and initially open-coded by the first author. A hybrid of conventional and directed content analysis was used to organize data into themes. ${ }^{15}$ Congruent with qualitative techniques for enhancing validity of findings, we checked to ensure that identified themes were consistent across sites and participant professional roles, and that contradictory or minority viewpoints were also considered in developing our analysis. Review of coding reports and discussion of interpretation occurred iteratively with the last author, and preliminary themes were also discussed on periodic calls with the full author team, which includes faculty at two of the CoEPCE sites. The Veterans Health Administration determined this work to be a quality improvement activity, with a waiver of informed consent.

\section{RESULTS}

Thirty-two non-faculty staff members from the five CoEPCEs volunteered for interviews (17 RNs, 4 Clerical Associates, and 11 Clinical Associates; see Table 1). Interviews occurred between May and December 2016 and lasted between 15 and $45 \mathrm{~min}$.

Our analysis of interviews highlighted six themes describing staff perspectives in working with interprofessional trainees (see Fig. 1). Four of these reflected positive impacts from interprofessional education efforts by engaging staff into the program's efforts (grouped in Fig. 1 as "engaging experiences"); in contrast, ambiguous or negative staff experiences were often described as being the result of conflict with or disengagement from the educational program's goals (grouped as "disengaging experiences"). As Fig. 1 reflects, while experiences that engaged participants in the educational efforts often resulted in overlapping perceived benefits, the tensions which staff experienced from duties that pulled them in directions away from the CoEPCE goals did not overlap and had clear boundaries.

Staff described numerous benefits from working with trainees in an interprofessional learning environment. We identified four different, though often intertwined, benefits from this engagement: (1) feeling valued as teachers and having opportunities as learners; (2) experiencing increased shared team leadership; (3) enjoying "fresh faces" and ongoing change; and (4) having a sense of contributing to the team-based future of health care. However, staff also noted two ways in which their work roles created tensions that pulled them away from others on the educational team: (1) management of a desire for continuity (both among patients and staff) sometimes challenged by CoEPCE practices; and (2) unsustainable demands on staff time created by competing priorities between education and the PCMH model.

\section{Integrating Experiences of PCMH Staff Members}

Feeling Valued as Teachers and Having Opportunities as

Learners. Just as the CoEPCEs stress a model of education in which individuals learn from and with each other, a crucial tenet of interprofessional education, staff described opportunities to learn and teach, often occurring simultaneously.

"I always think that everyone, no matter what position they hold here, has something to give to the trainee. I think from the LPN, the RNs, the social workers, I think we are all spokes in a wheel, and we are all important to help the trainee make it as good as an experience as possible. I take that as a responsibility, yes I do." Clinical associate 1, Site 5Related to this, staff described feeling valued by others for what they contributed to imparting skills and knowledge of clinical practice to trainees. 
Table 1 Participants by position and site

\begin{tabular}{|c|c|c|c|c|c|c|c|}
\hline Staff interviews & Role $^{\text {a }}$ & Site 1 & Site 2 & Site 3 & Site 4 & Site 5 & Total by position \\
\hline Nurse Care Manager & Registered nurse & 0 & 4 & 5 & 5 & 3 & 17 \\
\hline Clinical Associates & Licensed practical/vocational nurses & 1 & 0 & 5 & 2 & 3 & 11 \\
\hline Clerical Associates & Administrative clerk, medical support assistant & 2 & 0 & 2 & 0 & 0 & 4 \\
\hline Total by site & & 3 & 4 & 12 & 7 & 6 & 32 \\
\hline
\end{tabular}

${ }^{a}$ Precise definition of roles vary by site but all guided by VA's PCMH Model $(P A C T)^{11,} 16$

“...it's extra work when you work with trainee, but it's also a blast because they trust your skills, you're not just like, "Oh, you're just a nurse."... when somebody['s] with a doctor consulting, asking you questions, you feel like your work is valuable. You feel that you are being appreciated..." RN 1, Site 3

This simultaneous learning and teaching were described as empowering.

"Working with trainees empowers knowledge, you know I've gained some insight about them as they're working here in the clinic and as they are learning here, I'm also learning from them, you know." Clerical associate 1 , Site 1

Staff members described adapting to trainees' varying learning styles. When asked what skills are needed to work with trainees, a clinical associate stated:

"Honestly, patience and being able to teach properly. Being able to, as quickly as you can, identify the way the person learns and then teach them that way....you need to be able to recognize what kind of personality you have and what type of educator you are." Clinical associate 2 , Site 4
Explicitly interprofessional learning exercises for trainees often intentionally included staff participation, creating another venue for their engagement. For example, several RNs reflected positively on their role in patient care conferences ${ }^{17}$ that most CoEPCEs include in their curriculum:

"I am part of that [conference]. They consider my expertise as a nurse, the social worker, and other residents from other teams will be there, too. And the primary care provider who's going to be presenting it... we will be talking and getting the information and make up a plan, how to deal with this patient...I really like that. I really like to be part of everything." RN 1, Site 3

Notably, for long-time staff, the educational clinic provided a pathway for life-long learning:

...I mean they [trainees] have a lot of questions for us, too, and so it's, I mean, like working with the residents in the COE, they get to teach us, that's something we can figure out together, they're so new, so they're like willing to learn, right, and so like, I have over 20 years of experience as an RN..." RN 3, Site 1 Time and other constraints sometimes limited staff opportunities to engage as much as they would like.

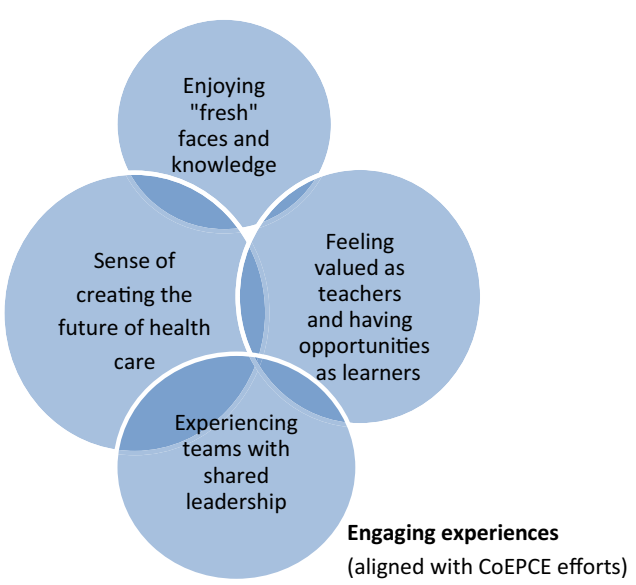

Disengaging experiences

(in tension with CoEPCE efforts)
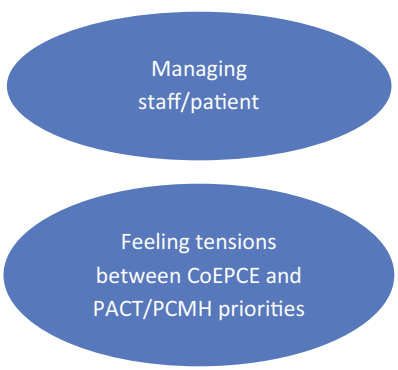

Fig. 1 Engaging and disengaging experiences of staff in five interprofessional primary care training clinics 
"So for my end, the learning that happens with the [interprofessional PACT team], that's usually in the admin building. But a lot of the education that goes with the learners doesn't really happen here.... we don't see that, we're not part of that. We could say, "Hey, I want to come" ... our learners will do a presentation, that's part of their thing. When I know it's one of our learners, the NP resident's doing it, I will go and see that. And then there are times when they present difficult patients, and we're invited to go if we want, but then we have to walk to the admin building ..." RN 4, Site 5

Experiencing Teams with Shared Leadership. CoEPCE clinics often intentionally practiced more distributed team leadership among the professions, such tools such as enhanced huddles. ${ }^{18}$ Staff interviews suggested a spillover effect for the entire care team, not just trainees in experiencing more distributed leadership and decisionmaking.

“...[here it is not] like you're just the front desk person, you're just the LVN... [or] 'I'm the MD and I'm in charge'- I definitely don't get that sense....Because for me, whether you're the front desk LPN, or, in the end it doesn't matter, you guys are a team. And you do whatever it takes." Clinical associate 4, Site 3

Others noted that the way huddles were structured by CoEPCE leadership helped them to speak up more often and feel more involved with the group.

"I've always been shy and quiet and then, but Dr. [CoEPCE director] is really good at training all of us to speak up, and she requires that everyone speaks up, including during huddles and also weekly we have to be approaching our trainees, our own trainees in the PACT teams to talk to them about the patients that they're going to see next week, so you really have to approach them and talk to them." Clinical associate 5, Site 3

Personal aspects of huddles and other interactions were also frequently cited as key to improved team communication.

"...one thing we do in the huddles, we do talk about personal things, 'how is your weekend,' just to make us feel a little bit more comfortable with each other. Once you feel more comfortable you feel like you can approach the person better, and you just get to know that person better, which makes working with that person a lot easier, too." Clerical associate 2, Site 3
Enjoying "Fresh" Faces and Knowledge. Participants acknowledged that working with trainees created more work, yet they derived pleasure from interacting with early career professionals and were stimulated by the "fresh" knowledge imparted to trainees in the clinical teaching environment. Multiple participants used the word "fresh" in describing their teammates and experiences, while others used similar terms. One RN with decades of experience reported:

"[Working with trainees] is something that I love to do, because it keeps me on my game, in that they are enthusiastic and bright and come in with all the latest and greatest in medicine. ...keeps me interested and enjoying my job, which is how I have managed to stay in it for so long [laughs]." RN 6, Site 3

Being in a learning environment created more opportunities, like those already mentioned, to teach and to learn:

I like watching them develop. Helping them develop. I like the change... you know...every year we get a new group coming in, and then watching them develop professionally. And then coach them a little bit on what works...." RN 7, Site 2

Sense of Creating the Future of Health Care. Some staff shared a sense that through sharing their knowledge with trainees, they were playing an important role in setting new norms of team-based behavior that would be disseminated by trainees when they went on to new positions.

"...the RN, the [Clinical Associate], that interacts the most with them [trainees] is grounding for the new young, youthful, educated, highly educated physicians that come in so that we can give them that base, that they have not yet gotten, but they will get." RN 6, Site 3

"It's an opportunity to get to know these providers before they've really fully developed their own practice, and I think going over the team initiatives and the team building and highlighting the importance of sharing in the work and developing the blend within the team that no one person in the team can do the work alone, and that you're going to enhance the patient care, and you're working smarter and not harder by spreading the work load among the people that's appropriate within everyone's unique scope of practice... you can target people, honestly, before they get too set in their ways and in their practice, and try to identify how you can work collaboratively." RN 5, Site 4 


\section{Experiences that Disengaged PCMH Staff Members from Educational Goals}

Managing Continuity Desires. The goal of teaching teambased care meant that CoEPCE visits frequently allowed patients to see more than one provider in a single visit. Some CoEPCEs had practice partner models where trainee providers rotated on a monthly basis, so that patients may be scheduled with a partner provider who they had not met before. As the members of the team most often responsible for arranging appointments and checking patients in, staff often felt a need to manage patient's expectations of seeing the same provider every time, and felt that this responsibility landed most heavily on them. Even when staff saw themselves as ambassadors of a team-based approach, they frequently empathized with patients' reasons for wanting provider continuity.

"I think the patients have a difficult time, also, because they, even though you tell them that this is a teaching facility, their expectation is always that they are going to see the same person, which is not the case... I think it's challenging... [in this clinic] to keep consistent for the patients, so that's he's not feeling like he has to repeat his story to multiple people. I think that's really challenging for the patient and I feel that for them." Clinical associate 1, Site 5An RN described how they dealt with this tension and played a role in patient education on team-based care:

I explain to them- you're gonna get excellent care with them. The new resident that comes in has access to their notes and their records and you really wouldn't have to explain everything all over again. And if you feel comfortable to stay with the resident I recommend you stay with the resident- but if you're adamant on changing then that's your option as well. I think a lot of them appreciate having a resident because they're more inquisitive, I think. RN 9, Site 4

Some staff also acknowledged wanting more continuity with "their" patients as well. Since team members were shared between multiple trainee providers at any given time, many staff felt they had less time to collaborate with each provider compared with the model of working with one full time provider and their panel of patients. This was particularly noted by Nurse Care Managers (RNs), who in the VA's PCMH model have an expanded care role that was described as difficult to deliver for the number of providers associated with multiple trainee providers (and possibly the challenge of faculty availability in any teaching clinic):

"I had worked for about 3-4 years in a non-trainee clinic and... I think I got to know my patients better in my teamlet, my panel, because the provider was there all the time [in that clinic], I was there all the time, they could come to us and, you know, with their needs, and have a relationship. Here, it's patient has a problem, provider isn't in, resident isn't in clinic for the next four weeks, we have to go to another one, and we have to, I don't know, it's just more fragmented... loosens that whole $[\mathrm{PCMH}]$ model because the patients end up getting scheduled to another random provider anyway, a lot of times". RN 8, Site 2

\section{Staff Capacity Stretched by Competing Priorities Between} Education and Patient Care in the PCMH Model. Staff recounted juggling competing priorities during the simultaneous implementation of the PACT and CoEPCE initiatives. Discussion of specific CoEPCE learning practices that were also clinical practices, such as interprofessional panel management ${ }^{19}$, illustrated these competing perspectives. One $\mathrm{RN}$ felt that they needed to prioritize what the provider needed to deliver care: "when I do panel management, I put the CoE down here, I put my PACT provider up here." (RN 2, Site 5) indicating that PACT takes priority over CoEPCE efforts. Additionally, this RN did not want trainees developing the expectation that staff would be supporting them so extensively in panel management once they finished their training, not only because they did not have time to do this but also "they should be doing their own panel management, right?"

As referenced earlier, PACT team members often were unable to attend all CoEPCE meetings, trainings, and huddles due to time constraints. In response, some prioritize PACT or CoEPCE team meetings, with the result of misaligned messages because there are few occasions where everyone meets to align vision.

“...there's really no formal setting [now] where all COEs [staff] getting together anymore so sometimes I think messages get blurred because even if it was a once a month thing, some kind of joint setting, might help because... when you hear something second, third, fourth hand, it's not exactly the same." RN 5 , Site 4

\section{DISCUSSION}

With non-faculty health professionals, including clerical and clinical associates, making up $60 \%$ of the health care workforce, ${ }^{20}$ more attention to their experiences and contributions to a team environment is warranted. For example, clerical associates are often not considered when thinking of broad system changes, but Solimeo found that leveraging and investing in these staff members may improve patient experiences, including providing a sense of continuity. ${ }^{21}$ This may be particularly true in an academic clinic where trainee turnover reduces continuity with primary care providers. 
Our findings support continued acknowledgement that nonfaculty staff are integral to the learning experiences of trainees, ${ }^{9,13}$ and confirm that the perspectives of staff members who work directly with patients are critical to successful trainee learning opportunities, such as the interprofessional case conferences adopted by the CoEPCEs. ${ }^{22}$ There has been a call to expand the concept of medical educators beyond the traditional physician preceptor, ${ }^{23}$ and our findings suggest that recognizing and supporting the educational role that primary care team members play partially answers that call. Recognizing this role by providing resources to formalize and fully integrate staff into educational experiences may contribute to better job satisfaction for staff, which in turn may positively influence outcomes for trainees and patients.

Findings from another study from our team found that patient outcomes were similar, and in some cases slightly improved, over comparable resident clinics ${ }^{24(B L I N D E D ~ F O R ~}$ REVIEW), providing evidence that interprofessional teambased learning is worthwhile. The challenge of meeting patient expectations of continuity of care within team-based models has been observed, ${ }^{25,}{ }^{26}$ however. CoEPCEs intentionally adopted a model of continuity that was team-based rather than dyadic, ${ }^{27}$ recognizing this may be in tension with existing patient expectations. Staff in our study experienced this tension, as well as a sense of potential conflict between the "patient centered" care at the core of the PCMH model and the needs of clinical learners. Our findings suggest that staff have a critical role to play in communicating to patients the benefits of team-based care, and that it is important for health care facilities to educate staff, and not just trainees or PCPs, to be able to do this well.

With appropriate recognition of the extra demands on time that may be created, our findings demonstrate that the presence of (interprofessional) learners can enhance work fulfillment for care team staff. The presence of learners impacts differently the work of RNs (who are more responsible for continuity and care coordination) and other team members (who deal with scheduling and access challenges). Recognizing the distinct role-determined needs of team staff may generate insights to improve team function and dynamics. Attention to the local context can help prioritize which activities, such as formal educational or QI projects, outside of direct patient care are easiest or most productive for team staff to participate in. Obtaining ongoing and open-ended feedback from staff, such as in this paper, may also be crucial to ensuring the educational program is well-embedded into the clinical context.

This study has some limitations. Our convenience sample relied on staff to be willing and available on the days that our evaluation team was able to conduct site visits. Site leadership and the evaluation team attempted to let all team staff working the days of the site visits know about the opportunity to be interviewed, but scheduling interviews during regular clinic duty hours limited many staff members' ability to participate. Although participants were assured that information provided to the evaluation team would not be reported to site leadership in any personally identifiable way, employees may have had privacy concerns that limited participation. These factors may have introduced biases into our participant sample and thus our findings.

\section{CONCLUSIONS}

Our analyses suggest that efforts to redesign academic primary care clinics to increase interprofessional collaboration and education have spillover effects for RNs and clerical/clinical associates. Innovations that the CoEPCEs developed, including interprofessional case management, population health panel management, and enhanced huddles, benefited staff by providing structure for their inclusion and recognition in a team environment. It is possible to build infrastructure for inclusion and recognition of non-faculty staff as key members of the team in settings without an interprofessional emphasis, suggesting these findings translate to other clinical team environments. Staff members reported enjoying and feeling engaged in education activities but struggled to participate due to disengaging factors such as other demands on their time. Teaching and guiding trainees in the clinic environment is a key function for staff, and despite resulting in more work, most participants reported great satisfaction in this role. Part of their satisfaction came from a sense of contributing to the future of health care by shaping new graduates. Challenges for staff in these clinics included managing continuity of care for patients and easing patient concerns about provider turnover and managing competing priorities when the needs and demands of PACT conflicted with those of the CoEPCEs. Primary care leaders have a role to play in acknowledging these tensions and providing space and resources for staff to propose possible solutions. Future research should identify and test locally developed strategies for enhancing staff ability to participate. Recognizing the teaching capacity that nonfaculty staff contribute to the training clinic and easing challenges to engagement in educational efforts may play a key role in retaining valuable staff. Taken together, our findings suggest that expanding opportunities for all care team members to contribute to the learning context is likely to enhance learning and satisfaction not just for formal learners but for the staff team members who are equally part of an evolving paradigm of health care delivery in the USA.

Corresponding Author: Summer Newell, PhD, MPH; Center to Improve Veteran Involvement in Care (CIVIC), VA Portland Healthcare System Portland, OR, USA (e-mail: summer.newell@va.gov).

Funding Information Quality Enhancement Research Initiative (QUERI) and the Office of Academic Affiliations, U.S. Department of Veterans Affairs.

\section{Compliance with Ethical Standards:}

Conflict of Interest: All authors declare that they do not have a conflict of interest. 
Disclaimer: The views expressed in this article are those of the authors and do not necessarily represent the views of the U.S. Department of Veterans Affairs.

\section{REFERENCES}

1. Margolius D, Bodenheimer T. Transforming primary care: From past practice to the practice of the future. Health Aff. 2010;29(5):779-784

2. Bitton A, Pereira AG, Smith CS, Babbott SF, Bowen JL. The EFECT framework for interprofessional education in the patient centered medical home. 2013;1(3-4):63-68.

3. Wilkins T, Yoo W, Gillies RA, et al.Patient-centered medical home status and preparedness to assess resident milestones: A CERA study. PRiMER. 2018;2:https://doi.org/10.22454/PRiMER.2018.710280.

4. Kozakowski SM, Eiff MP, Green LA, et al. Five key leadership actions needed to redesign family medicine residencies. Journal of graduate medical education. 2015;7(2):187-191

5. Ladden MD, Bodenheimer T, Fishman NW, et al. The emerging primary care workforce: Preliminary observations from the primary care team: Learning from effective ambulatory practices project. Acad Med. 2013;88(12): 1830-1834.

6. Brandt BF. Interprofessional education and collaborative practice: Welcome to the "new" forty-year old field. The Advisor. 2015:9-17.

7. Bodenheimer T, Ghorob A, Willard-Grace R, Grumbach $\mathbf{K}$. The 10 building blocks of high-performing primary care. Ann Fam Med. 2014;12(2): 166-171.

8. Bowen JL, Schectman G. VA academic PACT: A blueprint for primary care redesign in academic practice settings. VA Offices of Primary Care and Academic Affiliations. 2013

9. Clay MA, 2nd, Sikon AL, Lypson ML, et al. Teaching while learning while practicing: Reframing faculty development for the patient-centered medical home. Acad Med. 2013;88(9):1215-1219.

10. Office of Academic Affiliations, US Department of Veterans Affairs. Academic year 18-19 statistics: Health professions trainees. https:// www.va.gov/OAA/docs/OAA_Statistics_2019.pdf. Accessed December, 2019, .

11. Rosland AM, Nelson $\mathbf{K}$, Sun $\mathbf{H}$, et al. The patient-centered medical home in the Veterans Health Administration. Am $J$ Manag Care. 2013;19(7):e263-72.

12. Office of Academic Affiliations, US Department of Veterans Affairs. VA centers of excellence in primary care education, request for proposals. https://www.va.gov/OAA/docs/CoEPCE_Stage_1_RFP.pdf. Updated 2010. Accessed April 2, 2020.
13. Gilman SC, Chokshi DA, Bowen JL, Rugen KW, Cox M. Connecting the dots: Interprofessional health education and delivery system redesign at the veterans health administration. Acad Med. 2014;89(8):1113-1116.

14. Harada ND, Traylor $\mathbf{L}$, Rugen $\mathbf{K W}$, et al. Interprofessional transformation of clinical education: The first six years of the veterans affairs centers of excellence in primary care education. Journal of interprofessional care. 2018:1-9.

15. Hsieh H, Shannon SE. Three approaches to qualitative content analysis. Qual Health Res. 2005;15(9):1277-1288.

16. Klein S.The veterans health administration: Implementing patientcentered medical homes in the nation's largest integrated delivery system. Commonwealth Fund Washington, DC; 2011.

17. Weppner WG, Davis K, Sordahl J, et al. Interprofessional care conferences for high-risk primary care patients. Academic Medicine. 2016;91(6):798-802.

18. Shunk R, Dulay M, Chou CL, Janson S, O'Brien BC. Huddle-coaching: A dynamic intervention for trainees and staff to support team-based care. Acad Med. 2014;89(2):244-250.

19. Kaminetzky CP, Beste LA, Poppe AP, et al. Implementation of a novel population panel management curriculum among interprofessional health care trainees. BMC medical education. 2017;17(1):264.

20. Demo DH, Fry D, Devine N, Butler A. A call for action: Advocating for increased funding for the allied health professions: ASAHP leadership development program. J Allied Health. 2015;44(1):57-62.

21. Solimeo SL, Stewart GL, Rosenthal GE. The critical role of clerks in the patient-centered medical home. Ann Fam Med. 2016;14(4):377-379.

22. King IC, Strewler A, Wipf JE, et al. Translating innovation: Exploring dissemination of a unique case conference. Journal of Interprofessional Education \& Practice. 2017;6:55-60.

23. Gonzalo JD, Chang A, Wolpaw DR. New educator roles for health systems science: Implications of new physician competencies for U.S. medical school faculty. Acad Med. 2019;94(4):501-506.

24. Edwards ST, Hooker ER, Brienza R, et al. Association of a multisite interprofessional education initiative with quality of primary care. JAMA Network Open. 2019;2(11):e1915943-e1915943.

25. Feifer C, Mora A, White B, Barnett BP. Challenges to improving chronic disease care and training in residencies. Academic Medicine. 2006;81(8):696-701.

26. Keirns CC, Bosk CL. Perspective: The unintended consequences of training residents in dysfunctional outpatient settings. Academic Medicine. 2008;83(5):498-502.

27. Brienza RS, Zapatka S, Meyer EM. The case for interprofessional learning and collaborative practice in graduate medical education. Academic Medicine. 2014;89(11):1438-1439.

Publisher's Note: Springer Nature remains neutral with regard to jurisdictional claims in published maps and institutional affiliations. 